\title{
The Meaning of Semiochemicals to the Design of Self-Organizing Systems
}

\author{
Holger Kasinger, Bernhard Bauer \\ Department of Computer Science \\ University of Augsburg \\ 86135 Augsburg, Germany \\ \{kasinger, bauer\}@ds-lab.org
}

\author{
Jörg Denzinger \\ Department of Computer Science \\ University of Calgary \\ Calgary (AB), Canada T2N $1 N 4$ \\ denzinge@cpsc.ucalgary.ca
}

\begin{abstract}
In biology, many organisms coordinate their interactions in a self-organizing and emergent way solely by means of indirect communication based on chemical substances. These so-called semiochemicals comprise pheromones, mediating the coordination between organisms of the same species, as well as allelochemics, such as allomones, kairomones, synomones, and apneumones, mediating the coordination between organisms of different species. In order to exploit this biological diversity for the engineering of well adapted coordination mechanisms for self-organizing emergent multiagent systems, the micro- and macroscopic effects of the different types of semiochemicals have to be better understood. In this paper, we analyze these effects and based on that provide design guidelines that identify appropriate types of digital semiochemicals to use for the interactions between agents on the local level in order to achieve certain required effects on the global level. We apply these guidelines within an existing engineering methodology and present as a result an iteratively refined model of a decentralized coordination mechanism well adapted for the solution of pickup and delivery problems by self-organizing emergent multiagent systems.
\end{abstract}

\section{Introduction}

Future computer systems will be characterized by context-awareness, openness, locality in control, and locality in interactions [30]. They will contain a multiplicity of autonomous elements whose actions and interactions cannot be controlled by a single element. The system elements rather have to interact with each other on the local level in order to fulfill system-wide requirements on the global level. Thus, the elements have to be capable of coordinating their activities in a self-organizing and emergent way.

An essential key for these systems hence are appropriate mechanisms enabling the required self-organizing coordi- nation. Beside other sources of inspiration for such decentralized coordination mechanisms (DCMs), e.g. physics, economics, human societies, or social science that inspired mechanisms as presented in $[8,11,19,28]$ for instance, biology serves as the major one (see [1,9]). Living organisms achieve a self-organizing coordination by relying on different mechanisms of communication based on radiational (light perception or visual), mechanical (tactile or auditory), or chemical (gustatory or olfactory) stimuli.

The communication by chemical - more precisely olfactory - stimuli thereby is one of the most universally employed mechanisms in biology [17]. The chemical stimuli that convey information between organisms are termed semiochemicals [20]. They are divided into two groups: pheromones that mediate intraspecific interactions, i. e. between organisms of the same species, and allelochemics that mediate interspecific interactions, i.e. between organisms of different species. Both groups can be further subdivided into multiple subgroups.

Although the biological utilization of pheromones as chemical messengers has inspired various DCMs in the recent past, e. g. [3, 23, 25], the diversity of semiochemicals is promising for the identification and exploitation of new coordination paradigms, e. g. [15], as well as the refinement of already existing DCMs. For this purpose, we adopted the general principles behind the self-organizing coordination by semiochemicals in biology and developed a model for digital semiochemical coordination (DSC) [16] as a general basis. This high-level model defines a coarse-grained architecture of self-organizing emergent multi-agent systems (MASs) that interact by digital semiochemicals. Thus, it allows for any combination of digital semiochemicals in order to enable the best possible adaptation of fine-grained DCMs to the specific requirements of individual problems.

However, with regard to the engineering of such selforganizing emergent MASs and the appropriate utilization of the different types of semiochemicals, their effects on the local (microscopic) level as well as on the global (macroscopic) level have to be better understood. More precisely, 
guidelines have to be provided, which types of semiochemicals to use for the agents' interactions on the local level in order to achieve required effects on the global level. This is a nontrivial task, because in general the macroscopic properties of a self-organizing emergent MAS are not reducible to the microscopic behavior of the single agents (non-reductionism).

In order to derive the necessary guidelines, we again take advantage of biology: Any local interactions between living organisms or species in an ecosystem establish a global relationship between the interacting organisms. These relationships can be considered as macroscopic effects emerging from the microscopic behavior of the organisms. The microscopic effects of the relationships in turn can be beneficial, neutral, or harmful to the involved organisms. In this paper, we first survey these macroscopic relationships along with their microscopic effects on the involved organisms in detail (2.1). We afterward analyze the microscopic effects of the different types of semiochemicals (2.2). Based on the comparison of the effects we link the two levels (2.3), which provides first guidelines, which type of digital semiochemical to use for the interactions between agents on the local level in order to realize required effects on the global level. We apply these derived guidelines within an iteration-based engineering methodology [5] (which is described in Section 3) and present as a result an iteratively refined model of a DCM well adapted for the decentralized solution of pickup and delivery problems (PDPs) by a self-organizing emergent MAS (Section 4). Finally we present conclusions and an outlook on future work (Section 5).

\section{Design guidelines for systems based on dig- ital semiochemical coordination}

\subsection{The global level: macroscopic rela- tionships between living organisms}

In general, one distinguishes two different types of interactions between living organisms: intraspecific interactions between organisms of the same species and interspecific interactions between organisms of different species. Both types result in different relationships between the interacting organisms and have either beneficial, neutral, or harmful effects on the organisms or species involved [18]. The level of benefit or harm however is continuous and not discrete.

Intraspecific relationships can be classified along the degree of sociality exposed, ranging from nonsocial to social:

- Nonsocial relationships emerge from the tendency of organisms to avoid the association with others and to disregard the welfare of the own species. The reason may be a negative or positive egoistic behavior of an organism. Negative egoistic behavior refers to forms of selfish interactions, if the outcome is harmful to the general welfare of the own species or detrimental to other organisms. Positive egoistic behavior instead refers to forms of selfish interactions, if the outcome is self-interested but not harmful to the general welfare of the own species or detrimental to other organisms.

- Social relationships emerge from the tendency of organisms to associate with others and to form social groups. The reason may be a reciprocal altruistic, altruistic, or selfless behavior of an organism. Reciprocal altruistic behavior refers to the conversion of egoistic interactions into altruistic interactions. It describes social or cooperative forms of interactions, in which one organism provides a benefit to another organism of the same species without expecting any payment or compensation immediately ("tit for tat"). Altruistic behavior refers to social forms of interactions that increase the fitness of another organism of the same species without any immediate or later increase of the own fitness. Selfless behavior refers to social forms of interactions that increase the fitness of another organism while decreasing the own fitness. The organism therefore follows a course of action that has a high risk or certainty of suffering or death, which could otherwise be avoided.

Interspecific relationships that emerge between organisms or populations of different species range from antagonistic to mutualistic:

- Antagonistic relationships emerge from the interactions between (organisms of) different species, in which one species benefits at the expense of another species. There are two types of antagonism: predation and parasitism. Predation describes a relationship where a predator organism feeds on another living organism. Parasitism describes a relationship, in which one species, the parasite, benefits from the interaction with the other species, the host, which is harmed.

- Amensal relationships emerge from the interactions between (organisms of) different species, in which one species impedes or restricts the success of the other without being affected positively or negatively by the other. Amensalism sometimes is further divided into competition and antibiosis. For example some higher plants, e.g. the black walnut, secrete substances, e.g. juglone, that inhibit the growth of - or kill outright nearby competing plants.

- Commensal relationships emerge from the interactions between (organisms of) different species, in which one species benefits and the other species is neither benefited nor harmed. For example, whereas a small crab 
enters the shell of a oyster as a larva and receives shelter while it grows, once fully grown it is unable to exit through the narrow opening of the two valves and remains within the shell, snatching particles of food from the oyster but not harming its unwitting benefactor.

- Mutualistic relationships emerge from cooperative interactions between (organisms of) different species that normally benefit both species. They can be thought of as a form of biological barter in which species trade resources, such as carbohydrates or inorganic compounds, or services, such as protection from predators. For example, plants are hosts for insects that visit and pollinate them or eat their fruit.

There also exist relationships that apply to both types of interactions, in which the fitness of one organism or species has absolutely no effect on that of the other organism or species (neutral relationships) or the fitness of both is decreased (termed synnecrosis). However, true neutralism is very unlikely and impossible to prove, whereas synnecrosis is a rare and necessarily short-lived relationship as evolution selects against it.

\subsection{The local level: microscopic effects of semiochemicals}

Semiochemicals are classified according to their function or effect in specific interactions [27]. The classification depends on the ecological context in which it is being considered. Because the functions of semiochemicals are not mutually exclusive [2], the same semiochemical can function in two or three interactions. Moreover, in many cases not a single semiochemical takes effect on its own but different connections in a precisely defined mixture.

In general, semiochemicals can be classified into the two major groups of pheromones and allelochemics [20]. A pheromone is defined as a chemical substance secreted by an animal to the outside that causes a specific reaction in another member or members of the same species (conspecifics). The perception of a pheromone may result in an immediate behavioral response (releaser pheromones) or a complex set of physiological responses (primer pheromones) that are simply set in motion by the initial perception. Releaser pheromones are usually classified by the type of behavior they evoke (according to [12]):

- Sex pheromones are compounds used in the process of bringing the sexes together for the purpose of mating. They are known to exist in social insects.

- Aggregation pheromones cause conspecifics to aggregate in a particular area, e. g. a food source or a suitable habitat.
- Home range pheromones mark an area within which an organism normally confines its activities. When this area or territory is defended against other organisms, the chemicals are called territorial marking pheromones.

- Dispersal or spacing pheromones cause the spacing between conspecifics to increase, and can thus reduce intraspecific competition. These pheromones may be used to prevent overcrowding of resources such as food, mates, egg-laying sites, and refugia.

- Alarm pheromones are used by many insects to communicate to conspecifics that a danger is present. Thereby the term 'alarm' is used to describe the wide variety of behavioral responses caused by any threatening situation.

- Trail pheromones are used by many insects, especially by ants, for orientation to food sources or new nest sites. The odor trail system is one of the most elaborated forms of communication in social insects.

- Surface pheromones include recognition pheromones, releasers of grooming behavior and secretions that stimulate food exchange. They are very important in social insect colonies and allow recognition of nest mates, kin, or even members of different castes.

An allelochemic is defined as a chemical significant to organisms of a species different from its source [27]. Allelochemics are divided into four subgroups: allomones, kairomones, synomones, and apneumones. The division is based on whether the emitter, the receiver, or both benefit in the interaction:

- An allomone is defined as a chemical substance, produced or acquired by an organism, which evokes in the receiver a behavioral or physiological reaction adaptively favorable to the emitter. For example, a plant emits allomones to deter herbivores.

- A kairomone is defined as a chemical substance, produced or acquired by an organism, which evokes in the receiver a behavioral or physiological reaction adaptively favorable to the receiver but not to the emitter. For example, secondary plant compounds help herbivores in finding plants to feed.

- A synomone is defined as a chemical substance produced or acquired by an organism, which evokes in the receiver a behavioral or physiological response adaptively favorable to both the emitter and the receiver. This group of allelochemics includes floral scents and nectars that attract insects and other pollinators and substances that play an important but often subtle role in symbiotic relationships. 


\begin{tabular}{|c|c|c|c|}
\hline Macroscopic intraspecific relationship & $\begin{array}{l}\text { Effect on source } \\
\text { of stimulus }\end{array}$ & $\begin{array}{l}\text { Effect on sink of } \\
\text { stimulus }\end{array}$ & Corresponding type of pheromone \\
\hline \multicolumn{4}{|l|}{ Nonsocial } \\
\hline - Negative egoistic & + & - & Territorial pheromone \\
\hline - Positive egoistic & + & 0 & Home range pheromone \\
\hline \multicolumn{4}{|l|}{ Social } \\
\hline - Reciprocal altruistic & + & + & Dispersal, trail, sex, surface pheromone \\
\hline - Altruistic & 0 & + & Aggregation pheromone \\
\hline - Selfless & - & + & Alarm pheromone \\
\hline
\end{tabular}

Table 1. Macroscopic relationships with corresponding microscopic effects and pheromone types

- An apneumone is defined as a chemical substance emitted by a nonliving or abiotic material, which evokes in the receiver a behavioral or physiological reaction adaptively favorable to the receiver, but detrimental to an organism of a third species that may be found in or on the nonliving material. For example, a parasite or predator is attracted by apneumones to nonliving substances in which it may find another organism, its host or prey.

For the group of allelochemics a differentiation between releaser and primer allelochemics is also possible, although not all allelochemics are further subdivided. Kairomones can be classified according to the function for the benefiting organism into the four groups foraging kairomones, enemyavoidance kairomones, sexual kairomones, and aggregation kairomones [24] (whose descriptions are comparable to the corresponding types of pheromones). Whereas this classification can be transferred to allomones as well (except aggregation), the transfer to synomones is difficult. In many cases, a synomone will have different ecological functions for the emitter and for the receiver, which allows no clear criteria for a classification [24]. Because the group of apneumones has not been used any further since it was proposed, this group became superfluous over the years.

\subsection{Linking the global to the local level}

Table 1 provides guidelines, which type of digital pheromone to use for the interactions between homogeneous agents (agents of the same type) on the local level in order to realize required effects on the global level. The effects on the interacting agents are denoted by ' + ' (positive case), '0' (neutral case), or '-' (negative case) based on the analysis in Subsection 2.1. The meaning of these effects depends on the addressed problem domain, e. g. for solutions that require a spacial movement or routing of agents a ' + ' may indicate an attraction toward the direction of the perceived pheromone whereas a'-' may indicate a repellent effect into the opposite direction, provided that the micro- scopic behavior of the agents reflects the corresponding biological behavior of the organisms as analyzed in Subsection 2.2. Analogously, Table 2 provides guidelines, which type of digital allelochemic to use for the interactions between heterogeneous agents (agents of different types) in order to realize required effects.

\section{An iterative engineering methodology}

The elaborated guidelines may principally be used within any engineering methodology for MAS. Although the engineering of MAS has put various methodologies forth, e.g. $[7,10,14,21,22]$, the engineering of selforganizing emergent MASs is still in its infancy. The reason is that these methodologies mainly focus on the engineering of the (microscopic) behavior on the local level, i. e. the agents along with their rules, their interactions, etc., without explicitly engineering the required (macroscopic) behavior on the global level [29].

In this section we describe an iterative engineering methodology [5] that explicitly focuses on the engineering of issues on the global level (specified by macroscopic properties) of self-organizing emergent MASs. Although the methodology is not full-fledged completed yet, it provides a good starting point for the engineering of DSC-based systems. The methodology is based on the Unified Process (UP) [13], an iteration-based, incremental software development process framework that may be customized for specific organizations or projects. Figure 1 shows an iteration within the customized UP with focus on the engineering of macroscopic behavior of self-organizing emergent MASs.

Each iteration of the UP typically includes the process disciplines requirements analysis, design, implementation, as well as verification and testing. With each iteration the self-organizing emergent solution becomes successively refined, with cyclic feedback from verification to design. The focus of each discipline is on how to address the desired macroscopic properties. In the following, each customized discipline is described in more detail according to $[4,5]$. 


\begin{tabular}{|c|c|c|c|}
\hline Macroscopic interspecific relationship & $\begin{array}{c}\text { Effect on source } \\
\text { of stimulus }\end{array}$ & $\begin{array}{l}\text { Effect on sink of } \\
\text { stimulus }\end{array}$ & Corresponding type of allelochemic \\
\hline \multicolumn{4}{|l|}{ Antagonistic } \\
\hline - Predatory & + & - & Foraging, enemy-avoidance allomone \\
\hline - Parasitic & - & + & Foraging, enemy-avoidance kairomone \\
\hline \multicolumn{4}{|l|}{ Amensal } \\
\hline - Competitive/antibiotic & 0 & - & Allomone (in general) \\
\hline \multicolumn{4}{|l|}{ Commensal } \\
\hline - Inwards directed & + & 0 & Sexual allomone \\
\hline - Outwards directed & 0 & + & Sexual, aggregation kairomone \\
\hline \multicolumn{4}{|l|}{ Mutualistic } \\
\hline - Resource-resource coupled & + & + & Synomone \\
\hline - Service-resource coupled & + & + & Synomone \\
\hline - Service-service coupled & + & + & Synomone \\
\hline
\end{tabular}

Table 2. Macroscopic relationships with corresponding microscopic effects and allelochemic types

The requirements analysis emphasizes an investigation of the problem with functional and non-functional requirements. After the requirements are known, the decision has to be made if the (mostly non-functional) requirements indicate that a self-organizing emergent MAS is suitable for the solution of the investigated problem. This decision may be based on problem characteristics and requirements such as the desired degree of autonomy, decentralized or distributed information availability, as well as the desired robustness, flexibility, and availability. This customized discipline furthermore pays attention to issues that typically lead to self-organizing emergent MASs with macroscopic behavior, such as ongoing and adaptively maintained requirements - the so-called self-* properties.

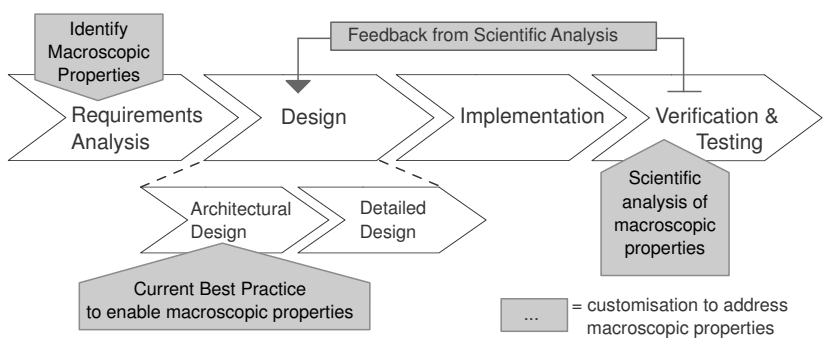

Figure 1. An iteration within the Unified Process customized for macro-scale issues [5]

The design emphasizes a conceptual solution that fulfills the identified requirements It is split into two phases: $a r$ chitectural design and detailed design. In early iterations of the engineering process the focus is more on the coarsegrained software architecture. The overall structure of the system as well as the interactions and relationships between software elements significantly influence the macroscopic properties of self-organizing emergent solutions. Therefore, knowledge and experience (design principles, reference architectures, DCMs) from existing best practice in engineering self-organizing emergent solutions has to be exploited. In later iterations the architecture converges more and more to a fixed structure and fine-grained design issues are resolved, i. e. mainly the microscopic behavior of agents.

The implementation realizes the previously specified (hardware and software) design in code. This requires no special customizations for macroscopic issues because the implementation is completely microscopic.

In early iterations of verification and testing the focus is more on the coarse-grained decisions with respect to the architecture of the solution. This includes an analysis of non-functional and macroscopic performance, a comparison of coordination mechanisms, parameter tuning, etc. In later iterations the focus is more on the details of the solution, i.e. setting and changing parameters to adaptively react to changing situations. This serves as important feedback for the correct adaptation of the design. Note, although the macroscopic behavior can still be specified formally, it is practically infeasible to formally verify or proof the correctness of the macroscopic behavior (see [4]).

\section{Engineering self-organizing emergent multi-agent systems based on DSC}

In this section we describe our experiences from the application of the elaborated guidelines of Section 2 within the iterative engineering methodology described in the last section to the design and refinement of a DCM well adapted for the decentralized solution of PDPs by a self-organizing emergent MAS. A PDP is a specialization of the General Pickup and Delivery Problem (GPDP) [26] and primarily 
comprises the transportation of objects, goods, or persons by vehicles from a set of pickup stations to a set of delivery stations along with the optimization (as best as possible) of an objective function, e. g. minimization of route length, minimization of total travel time, etc. All vehicles start and finish their routes in a central depot. Practical examples are e.g. freight transportation systems, courier services, repair services, emergency services, or express mail delivery as well as manufacturing systems. For lack of space we only describe the architectural and detailed design. Thus, at this point we assume that the requirements analysis executed before had identified a self-organizing emergent MAS to be suitable for the solution of such PDPs.

Altogether we executed four major iterations during the engineering process, whereas each major iteration included several minor iterations. The focus of the first to the third iteration was rather on the architectural design, i.e. on the system architecture and the different digital semiochemicals, whereas the focus of the fourth iteration was rather on the detailed design, i. e. on the internal behavior of agents. For every iteration we took test and verification results (extensive simulations and experimentations) of the respective prior iteration into account.

\subsection{First iteration}

The main issue of the first iteration was to identify an appropriate basic design pattern. The requirements we had allowed for a DSC-based DCM. If we had changed the requirements, other DCMs, e.g. gradient-field coordination [19], would have been suitable too. Our analysis of the problem identified three different types of agents participating in the solution: vehicle agents, (pickup/delivery) station agents, as well as a depot agent. Because the major interactions in such PDPs have to take place between the station agents and the vehicle agents, we started with the classification of this relationship.

Because vehicle agents provide a beneficial service to station agents (transportation of objects) and station agents usually provide beneficial resources (in form of a payment) to vehicle agents for this service, both agent types benefit in this interaction. According to the guidelines in Table 2, this indicates a service-resource coupled, mutual relationship between these agent types. Although the guidelines in this case recommend the use of synomones for the coordination on the local level, they do not recommend a specific DCM, which still has to be derived from Best Practice. Due to the requirement the pollination-inspired coordination (PIC) mechanisms [15] seemed to be appropriate. PIC is inspired by the pollination of plants by honey bees, which are attracted by synomones in form of fragrances emitted by the plants. The interest and benefit of plants (as pollenizers) in pollination is the accomplishment of their reproduction.
For the purpose of reproduction, an important prerequisite is the transfer of male gametes, the pollen grains, from the male reproduction organ of a plant's flower to the female reproduction organ of another flower. This usually requires a biotic pollinator, such as honey bees, to transfer the pollen grains. The interest and benefit of honey bees in pollination in turn is the maximization of the success in their own reproduction by optimal foraging. The latter is measured by (1) energy gains per time unit, which requires the detection of essential nutrients, (2) minimal time for flower detection, and (3) short handling time during the flower visits. Plants facilitate optimal foraging of their pollinators, if their behaviors attend to the transfer of their pollen grains. Therefore, plants provide food such as pollen or nectar as a form of reward for their visitors, but they are not that generous, as that pollinators can renounce the pollen grain transfer to other plants.

Because vehicle agents usually benefit from the return to the depot agent, e. g. for fueling or loading, and the depot agent on the other hand is neither positively nor negatively affected by the visit of vehicle agents, only the vehicle agents benefit in this interaction. According to the guidelines in Table 2, this indicates an outwards-directed commensal relationship on the part of the depot agent between these agent types. In this case the guidelines recommend the utilization of aggregation kairomones by the depot agents for the interactions. Because station agents and the depot agent do not affect each other directly, there is no need for any interactions between these two types.

These analyses finally led us to the design of the PIC model for PDPs, which is depicted in Figure $2^{1}$. Note, the model instantiates the DSC model (see [16]) but is not customized to a specific application domain yet. In this PIC model, a Pollenizer Agent (the later station agent), a Pollinator Agent (the later vehicle agent) and a Hive Agent (the later depot agent) are situated on a Location in a common Environment. Pollenizer agents belong to a certain Pollenizer Type, pollinator agents to a Pollinator Type, and hive agents to a Hive Type. The pollinator type is linked to the pollenizer type, such that pollinator agents can observe Synomones emitted by pollenizer agents. Moreover, the pollinator type is linked to the hive type, such that pollinator agents can observe Aggregation Kairomones emitted by the hive agent. Thus, different Semiochemical Collections composed of multiple of these Semiochemicalss guide a pollinator agent from its current location to suitable locations of a pollenizer agent or a hive agent.

Due to this bouquet of different semiochemicals managed by specialized Semiochemical Actions

\footnotetext{
${ }^{1}$ For lack of space, this figure represents the final model after the fourth iteration. Allomones and territorial pheromones represent model refinements resulting from later iterations and are not part of the basic model.
} 


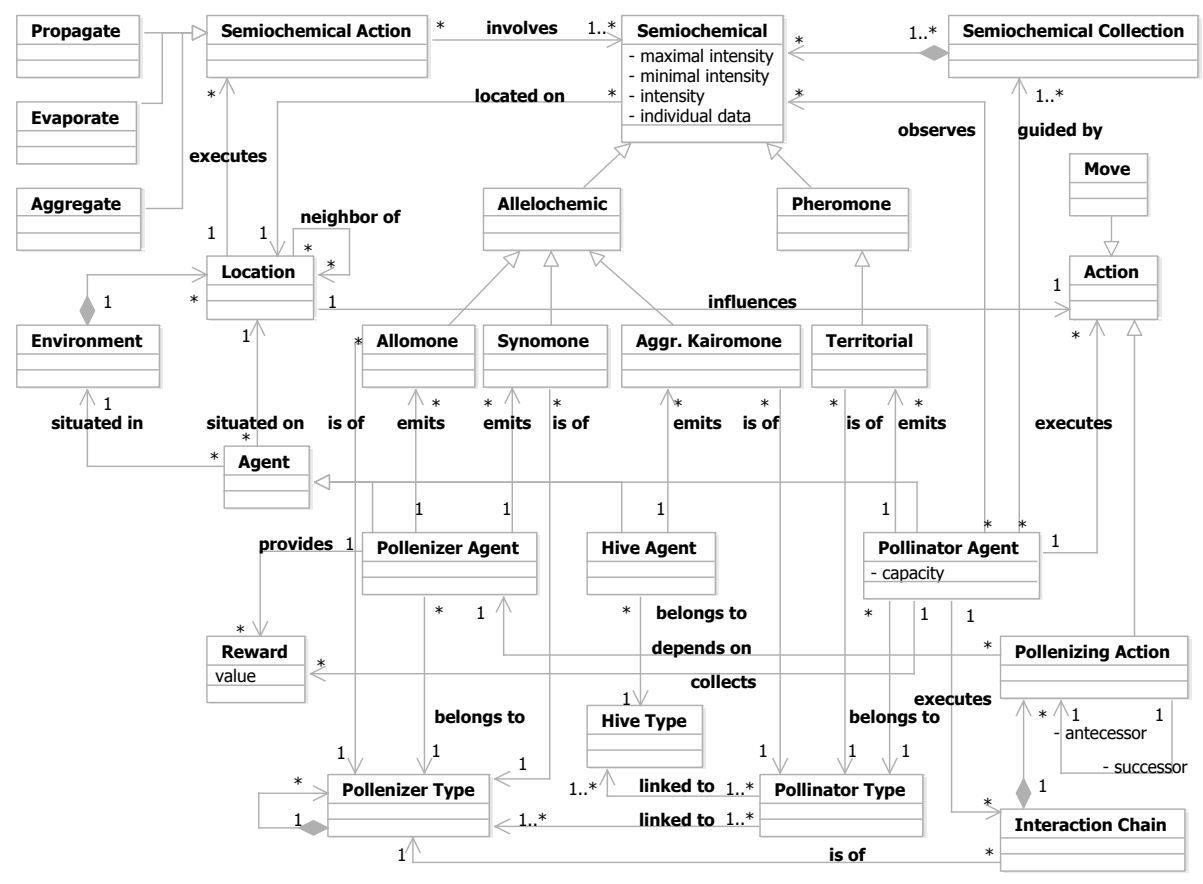

Figure 2. PIC model for the solution of PDPs involving four types of semiochemicals

of the locations in the environment, pollinator agents are able to Move through the environment according to the specific needs of pollenizer or hive agents. In order to fulfill these needs, pollinator agents may execute Interaction Chains comprising Pollenizing Actions (in this case pickup and delivery actions). A pollinator agent needs to visit at least one more pollenizer agent after the initial visit to the first pollenizer. An interaction chain of a pollinator agent thus represents an intended sequence of interactions with pollenizer agents that all need to be performed to fulfill a task the software engineer wants to achieve. A pollinator agent can be involved in several interaction chains, but we usually limit the number of "open" chains by a capacity.

A pollenizer agent provides a Reward with a variable value (representing the estimated value of a reward for a pollinator in biology) for each pollenizing action performed. The value of a reward $r$ provided by a pollenizer agent $e$ at time $t$ varies according to the following function:

$$
r^{e}(t):=r^{e}\left(t_{\text {init }}\right) \cdot w \cdot \delta, w= \begin{cases}t-t_{\text {init }}, & \text { if } t>t_{\text {init }} \\ 1, & \text { if } t=t_{\text {init }}\end{cases}
$$

where $t_{\text {init }}$ is the initialization time of $e$ and $\delta$ is a constant adjustment factor with $0<=\delta<=1$. This means that the longer a pollenizer agent waits for its pollination, the higher is the value of its reward, which makes the pollenizer more attractive to visit. The value of a reward hence is comparable to market prices: the lower the supply of pollinator agents, the higher the price a pollenizer agent pays for pollination. This value is encapsulated in the individual data of a synomone and thus made indirectly available to potential pollinator agents, which take it into account for their decisions. Similar to the increase of the reward value, the pollenizer agent also increases the initial intensity of synomones it emits, which enables a greater propagation area possibly covering more receivers.

Propagation algorithm The guidance of pollinator agents to the reward providing pollenizer agents and to the hive agent is supported by a kind of gradient field the allelochemics span around its emitters due to the propagation algorithm. Generally, if an arbitrary semiochemical $\mathrm{sem}^{e}$ of an emitting agent $e$ is propagated from a location $l_{\text {dep }}$ to a location $l_{\text {dest }}$, its intensity $s e m_{i}^{e}$ is decreased according to the following function:

$$
\operatorname{sem}_{i}^{e}\left(l_{\text {dest }}\right):=\operatorname{sem}_{i}^{e}\left(l_{\text {dep }}\right)-d\left(l_{\text {dep }}, l_{\text {dest }}\right)
$$

where $d\left(l_{\text {dep }}, l_{\text {dest }}\right)$ is the distance between these two locations, measured e.g. in meters, hops, etc. If at $l_{\text {dest }}$ no other semiochemical $\operatorname{sem}^{f}$ with $e=f$ of the same type (synomone or kairomone, as well as at the end allomone or pheromone) is currently present, $\mathrm{sem}^{e}$ will be stored at the end of the incoming connection to $l_{d e p}$, cloned, and its clones propagated on all outgoing connections to other 
neighboring locations depending on the applicable semiochemical action for this semiochemical type. If in contrast there is a $\operatorname{sem}^{f}, e=f$, stored at $l_{\text {dest }}$, the intensities of both instances will be compared. If $\mathrm{sem}_{i}^{e}>\mathrm{sem}_{i}^{f}$, then $\mathrm{sem}^{e}$ will replace $\operatorname{sem}^{f}$ and will be cloned as well as propagated, otherwise $\mathrm{sem}^{e}$ will be discarded. Hence there is always at most one instance of a semiochemical type of a certain emitter stored at one location. Semiochemicals of different emitters as well as different semiochemical types of the same emitter, however, can be stored in parallel on one location. A pollinator agent following a certain allelochemic thus will always be guided to the allelochemic's emitter on the shortest path, without the comparison of identical allelochemics.

\subsection{Second iteration}

This PIC model so far enables robust and flexible solutions despite dynamic changes. However, test results at the end of the first iteration revealed that in certain cases pollinator agents were misdirected by outdated synomones. Not until an unrefreshed synomone was evaporated, it ceased guiding pollinator agents to its emitting pollenizer agents, even if the needs of the pollenizer agent were already fulfilled. The higher the evaporation factor, the longer unrefreshed synomones remained in the environment resulting in a deterioration of the overall efficiency of the system.

Thus, in such cases the pollenizer agents have to keep further pollinator agents off from visiting. According to the guidelines in Table 2, this suggests a kind of antibiotic relationship between the pollenizer and pollinator agents. Pollenizer agents hence have to emit Allomones, which are similar to the synomones propagated through the environment. An approaching pollinator agent observing the outdated synomones of this pollenizer agent thus will be kept away due to the additionally observed allomones and look for other pollenizers.

\subsection{Third iteration}

Despite the efficiency improvements achieved by the model refinement in the second iteration, further test results revealed that in certain cases multiple pollinator agents were attracted to one pollenizer agent, although only one pollinator agent would have sufficed. Only the pollinator agent arriving first was successfully executing a pollenizing action, while the rest had to go away empty-handed. This kind of undesired swarm movement reduced the overall efficiency significantly, as these unsuccessful pollinators were missed at other locations.

Thus, in such cases pollinator agents have to force other pollinator agents that move in the same direction to switch to another direction. According to the guidelines in Table
1 , this requires a kind of nonsocial relationship between the pollinator agents, more precisely a negative egoistic behavior of pollinator agents. They hence have to emit Territorial Pheromones that are propagated in a small area only, including the information which pollenizer agent the emitting pollinator agent intends to visit. If a following pollinator agent with the same target observes such a pheromone, it thereby will switch to another synomone.

\subsection{Fourth iteration}

Due to the model refinement in the third iteration, the overall efficiency of the solution was satisfying enough and hence the system architecture was mainly fixed. Thus, the fourth iteration focused on the internal behavior of the agents. In this paper, we only describe the local decision mechanism of pollinator agents, although the behavior of pollenizer agents and the hive agent were designed too.

The local decision mechanism of any pollinator agent $p$ of pollinator type pot is very critical. The agent has to decide very quickly, only based on the locally observable information, which allelochemic, especially which synomone, to follow in order to act efficiently for itself but also for the entire system. This is exacerbated by the fact that $p$ is allowed to handle multiple interaction chains $I c$ in parallel up to its capacity (defined as $I c(p)<c a p$ ). However, $p$ is only allowed to handle one interaction chain $I c_{p t}$ of a certain pollenizer type $p t$ in parallel, i.e. $I c_{p t}(p)=\{0,1\}$. The pollenizer type of an interaction chain $I c_{p t}$ is determined by the pollenizer type of the pollenizer agent the first pollenizing action was executed with.

The decision mechanism first of all is based on the estimated utility $u\left(s_{p t}^{e}\right)$ of each observable synomone $s_{p t}^{e}$ emitted by $e$ of pollenizer type $p t$ on the current location, but also additionally regards any present allomone $a$ or territorial pheromone $p h$. The calculation of this utility is governed by the following policies:

- If p has not started an interaction chain I $c_{p t}$, yet, i.e. $I c_{p t}(p)=0$ :

- The longer the time $p$ follows $s_{p t}^{e}, t_{s_{p t}^{e}}$, the higher is the utility $u\left(s_{p t}^{e}\right)$. This is a tribute to the effort that resulted from following the gradient of $s_{p t}^{e}$ up to this location.

- The nearer $p$ is to the location of $e$, the higher is the utility $u\left(s_{p t}^{e}\right)$. Thus, nearer pollenizer agents are privileged compared to others.

- The higher the value of the provided reward by $e, r^{e}$, the higher is the utility $u\left(s_{p t}^{e}\right)$. Thus, pollenizer agents that already wait longer for its pollination are privileged. 
- If $p$ observes an allomone $a^{e}$ emitted by $e$, the moving to $e$ will have no more utility for $p$. Note, a pollenizer agent emits an allomone as soon as a pollenizing action by a visiting pollinator agent fulfills its needs.

- If $p$ observes a pheromone $p h_{e}^{o}$ of another pollinator agent $o$ that also intends to visit $e$ with $p \neq o$, for a certain amount of time $s_{p t}^{e}$ will have no more utility for $p$ and will be excluded from the utility calculation. If after this time $p$ still observes $s_{p t}^{e}$ but no more $p h_{e}^{o}, s_{p t}^{e}$ will be regarded again for calculation.

- If p has already started an interaction chain I $c_{p t}$, i.e. $I c_{p t}(p)=1$ :

- The utility $u\left(s_{p t}^{e}\right)$ has to be remarkable higher compared to the case $I c_{p t}(p)=0$.

- If $p$ observes an allomone $a^{e}$ emitted by $e$, the moving to $e$ will also have no more utility for $p$.

- If $p$ observes a pheromone $p h_{e}^{o}$ of another pollinator agent $o$ that also intends to visit $e$ with $p \neq o$, the utility $u\left(s_{p t}^{e}\right)$ depends on the amount of further suitable synomones $s_{p t}^{f}, e \neq f$, that can be observed on this location. If no more suitable synomones can be observed, the utility $u\left(s_{p t}^{e}\right)$ is calculated as described by the first policy in this case. If there is at least one more suitable synomone $s_{p t}^{f}$, again for a certain amount of time $s_{p t}^{e}$ will have no more utility for $p$.

These policies guarantee that the processing or even closing of interaction chains is prioritized over the starting of new interaction chains. The policies are incorporated into a utility function that has to be applied to every synomone $s_{p t}^{e}$ observed at the pollinator's current location:

$u\left(s_{p t}^{e}\right):= \begin{cases}b, & \text { if } I c_{p t}(p)=0 \wedge l\left(a^{e}\right)=0 \wedge l\left(p h_{e}^{o}\right)=0 \\ b \cdot \lambda, & \text { if } I c_{p t}(p)=1 \wedge l\left(a^{e}\right)=0 \\ & \wedge\left(l\left(p h_{e}^{o}\right)=0 \vee l\left(p h_{e}^{o}\right)>0 \wedge l\left(s_{p t}^{f}\right)>0\right) \\ 0, & \text { else }\end{cases}$

where

- $b$ is defined as $t_{s_{p t}^{e}} \cdot \alpha+\frac{s_{i \max }^{e}}{s_{i \max }^{e}-s_{i}^{e}} \cdot \beta+r^{e}(s) \cdot \gamma, s_{i^{\max }}^{e}$ is the initial intensity of $s_{p t}^{e}$, and $\alpha, \beta, \gamma, \lambda>=0$ are a adjustment factors

- $l\left(a^{e}\right)$ returns the amount of $a^{e}$ present at the location

- $l\left(p h_{e}^{o}\right)$ returns the amount of pheromones emitted by any pollinator $o \neq p$ that intends to visit $e$

- $l\left(s_{p t}^{f}\right)$ returns the amount of other suitable synomones present at the location
After calculating the utilities of all synomones observed on its current location, $p$ emits a pheromone $p h_{e}^{p}$ in order to indicate its intension to visit $e$, with $u\left(s_{p t}^{e}\right)$ is maximal, to possibly following pollinators, and moves to the location connected by the incoming direction of the synomone $s_{p t}^{e}$. If this is the location of $e, p$ then will either start a new interaction chain or execute the next (or even the last) action of any open interaction chain.

If no synomones are found at all, $p$ will either remain on its current location, search for other synomones by moving randomly, or follow the aggregation kairomones of the nearest hive agent $g$ (usually only one) of a linked hive type $h t$, depending on the application needs. Again, in order to decide, which kairomone to follow, $p$ estimates the utility $u\left(k_{h t}^{g}\right)$ of each observable kairomone $k_{h t}^{g}$ emitted by $g$ on the current location:

$$
u\left(k_{h t}^{g}\right):=\frac{k_{i_{\max }}^{g}}{k_{i^{\max }}^{g}-k_{i}^{g}}
$$

where $k_{i \max }^{g}$ is the initial intensity of $k_{h t}^{g}$. After this calculation, $p$ moves to the location connected by the incoming direction of $k_{h t}^{g}$. Note, to follow synomones has a higher priority than to follow kairomones. Thus, if $p$ observes appropriate synomones on its way to $g$, it will follow them.

\section{Conclusions and outlook}

In this paper we presented first design guidelines, which type of digital semiochemical to use for the interactions between agents on the local level in order to realize required effects on the global level. The guidelines were derived from an analysis of macroscopic biological relationships emerging from microscopic interactions between living organisms as well as of the microscopic effects of different types of semiochemicals. We applied these guidelines within an iterative engineering methodology to the design and refinement of a DCM well adapted for the decentralized solution of PDPs by a self-organizing emergent MAS. The engineering process demonstrated the usefulness of the guidelines, as they recommended the utilization of certain types of semiochemicals in order to choose a basic design pattern as well as to improve the overall efficiency of the system for the solution of PDPs. Experimental results satisfactorily showing these improvements can be found in[16].

Of course, regarding the engineering of self-organizing emergent MASs based on DSC there are still some open issues. First, software engineers require further guidelines, for which problems a DCM based on DSC is more suitable compared to other DCMs, such as e. g. [8, 11, 19, 28]. These guidelines may extend the work started in [6]. Second, although the presented guidelines for DSC recommend the use of certain types of semiochemicals, they do not recommend the use of specific design patterns yet. However, 
whereas for the group of pheromones a couple of design patterns already exist, appropriate design patterns are still missing for the group of allelochemics. To our knowledge, the PIC model is the only one. Third, the meaning of positive and negative effects on agents have to be clarified for other problem domains that do not require a spacial movement of agents, in order to broaden the applicability of DSC.

However, in our opinion the focus of future research in this area should be primarily on the identification of further design patterns based on semiochemicals. Therefore, the presented guidelines provide a valuable starting point.

\section{References}

[1] O. Babaoglu, G. Canright, A. Deutsch, G. A. D. Caro, F. Ducatelle, L. M. Gambardella, N. Ganguly, M. Jelasity, R. Montemanni, A. Montresor, and T. Urnes. Design patterns from biology for distributed computing. ACM Transactions on Autonomous and Adaptive Systems (TAAS), 1(1):26-66, September 2006.

[2] W. L. Brown, Jr., T. Eisner, and R. H. Whittaker. Allomones and kairomones: Transpecific chemical messengers. BioScience, 20:21-22, 1970.

[3] S. Brückner. Return from the Ant - Synthetic Ecosystems for Manufacturing Control. Phd thesis, Humboldt-Universität, Berlin, 2000.

[4] T. De Wolf. Analysing and Engineering Self-organising Emergent Applications. PhD thesis, Department of Computer Science, K.U.Leuven, Leuven, Belgium, May 2007.

[5] T. De Wolf and T. Holvoet. Towards a methodolgy for engineering self-organising emergent systems. SelfOrganization and Autonomic Informatics (I), Frontiers in Artificial Intelligence and Applications, 135:18-34, 2005.

[6] T. De Wolf and T. Holvoet. A taxonomy for self-* properties in decentralised autonomic computing. In M. Parashar and S. Hariri, editors, Autonomic Computing: Concepts, Infrastructure, and Applications, pages 101-120. CRC Press, 2007.

[7] S. A. DeLoach, M. F. Wood, and C. H. Sparkman. Multiagent systems engineering. Int. Journal of Software Engineering and Knowledge Engineering, 11(3):231-258, 2001.

[8] M. B. Dias, R. Zlot, N. Kalra, and A. Stentz. Market-based multirobot coordination: A survey and analysis. Proceedings of the IEEE, 94(7):1257-1270, July 2006.

[9] L. Gardelli, M. Viroli, and A. Omicini. Design patterns for self-organizing multiagent systems. In Proceedings of CEEMAS 2007, Leipzig, Germany, volume 4696 of LNCS, pages 123-132. Springer, 2007.

[10] P. Giorgini, M. Kolp, J. Mylopoulos, and M. Pistore. The tropos methodology: An overview. In F. Bergenti, M.P. Gleizes, and F. Zambonelli, editors, Methodologies And Software Engineering For Agent Systems. Kluwer Academic Publishing, 2004.

[11] D. Hales. Choose your tribe! - evolution at the next level in a peer-to-peer network. In Proceedings of ESOA 2005, Utrecht, The Netherlands, Revised Selected Papers, volume 3910 of LNCS, pages 61-74. Springer, 2006.
[12] B. D. Jackson and E. D. Morgan. Insect chemical communication: Pheromones and exocrine glands of ants. Chemoecology, 4(3-4):125-144, Sep 1993.

[13] I. Jacobson, G. Booch, and J. Rumbaugh. The unified software development process. Addison Wesley, 1999.

[14] T. Juan, A. R. Pearce, and L. Sterling. Roadmap: extending the gaia methodology for complex open systems. In Proceedings of AAMAS 2002, Bologna, Italy, pages 3-10. ACM, 2002.

[15] H. Kasinger and B. Bauer. Pollination - a biologically inspired paradigm for self-managing systems. Journal of International Transactions on Systems Science and Applications, 2(2):147-156, September 2006.

[16] H. Kasinger, J. Denzinger, and B. Bauer. Digital semiochemical coordination. Communications of SIWN, 4:133139, June 2008.

[17] T. Lewis. The elements and frontiers of insect communication. In T. Lewis, editor, Insect Communication, pages 1-27. Academic Press, London, 1984.

[18] W. Z. Lidicker, Jr. A clarification of interactions in ecological systems. BioScience, 29(8):475-477, Aug 1979.

[19] M. Mamei and F. Zambonelli. Field-Based Coordination for Pervasive Multiagent Systems. Springer Series on Agent Technology. Springer-Verlag, 2006.

[20] D. A. Nordlund and W. J. Lewis. Terminology of chemical releasing stimuli in intraspecific and interspecific interactions. Journal of Chemical Ecology, 2(2):211-220, 1976.

[21] A. Omicini. Soda: societies and infrastructures in the analysis and design of agent-based systems. In Proceedings of AOSE 2000, pages 185-193, Limerick, Ireland, 2001. Springer-Verlag New York, Inc.

[22] L. Padgham and M. Winikoff. Developing Intelligent Agent Systems: A Practical Guide. John Wiley \& Sons, 2004.

[23] L. Panait and S. Luke. A pheromone-based utility model for collaborative foraging. In Proceedings of AAMAS 2004, New York, USA, pages 36-43. IEEE Computer Society, 2004.

[24] J. Ruther, T. Meiners, and J. L. M. Steidle. Rich in phenomena-lacking in terms. a classification of kairomones. Chemoecology, 12(4):161-167, November 2002.

[25] J. A. Sauter, R. Matthews, H. V. D. Parunak, and S. A. Brückner. Performance of digital pheromones for swarming vehicle control. In Proceedings of AAMAS 2005, Utrecht, The Netherlands, pages 903-910. ACM, 2005.

[26] M. W. P. Savelsbergh and M. Sol. The general pickup and delivery problem. Transporatation Science, 29:17-29, 1995.

[27] R. H. Whittaker and P. P. Feeny. Allelochemics: Chemical interactions between species. Science, 171:757-770, 1971.

[28] Y. Xu, P. Scerri, B. Yu, S. Okamoto, M. Lewis, and K. P. Sycara. An integrated token-based algorithm for scalable coordination. In Proceedings of AAMAS 2005, Utrecht, The Netherlands, pages 407-414. ACM, 2005.

[29] F. Zambonelli and A. Omicini. Challenges and research directions in agent-oriented software engineering. Auton. Agents and Multi-Agent Systems, 9(3):253-283, 2004.

[30] F. Zambonelli and H. V. D. Parunak. Towards a paradigm change in computer science and software engineering: a synthesis. The Knowledge Engineering Review, 18(4):329342, December 2003. 